\title{
Nuclear de-excitation lines as a probe of low-energy cosmic rays
}

\author{
Bing Liu ${ }^{1,2,3,4}$, Rui-zhi Yang ${ }^{1,2,3 \dagger}$, and Felix Aharonian ${ }^{5,6,7}$ \\ ${ }^{1}$ Department of Astronomy, School of Physical Sciences, University of Science and Technology of China, Hefei, Anhui 230026, \\ China ${ }^{\dagger}$ yangrz@ustc.edu.cn \\ 2 CAS Key Laboratory for Research in Galaxies and Cosmology, University of Science and Technology of China, Hefei, Anhui \\ 230026, China \\ 3 School of Astronomy and Space Science, University of Science and Technology of China, Hefei, Anhui 230026, China \\ 4 Key Laboratory of Modern Astronomy and Astrophysics (Nanjing University), Ministry of Education, Nanjing 210093, China \\ 5 Dublin Institute for Advanced Studies, 31 Fitzwilliam Place, Dublin 2, Ireland \\ 6 Max-Planck-Institut für Kernphysik, P.O. Box 103980, D 69029 Heidelberg, Germany \\ 7 National Research Nuclear University MEPhI, Kashirskoje Shosse, 31, 115409 Moscow, Russia
}

January 12,2021

\begin{abstract}
Low-energy cosmic rays (LECRs) contribute substantially to the energy balance of the interstellar medium. They play also significant role in the heating and chemistry of gas, and, consequently, on the star formation process. Because of the slow propagation coupled with enhanced energy losses of subrelativistic particles, LECRs are concentrated around their acceleration sites. LECRs effectively interact with the ambient gas through nuclear reactions. Although these processes are energetically less effective compared to heating and ionization, they are extremely important from the point of view of nuclear de-excitation lines, which carry unique information about LECRs. We present results on production of de-excitation lines combining the numerical treatment of nuclear reactions using the code TALYS, with the propagation and energy losses of LECRs.
\end{abstract}

Key words. cosmic rays - $\gamma$-rays: ISM

\section{Introduction}

The energy density of Galactic cosmic rays $(\mathrm{CRs}), w_{\mathrm{CR}} \approx$ $1 \mathrm{eV} / \mathrm{cm}^{3}$, is comparable to the energy density contributed by the interstellar magnetic fields and thermal gas. CRs play an essential role in the process of star formation through the heating and ionization, initiating several crucial chemical reactions in the dense cores of molecular clouds (Papadopoulos 2010).

The flux and spectra of CRs inside the Solar System have been measured with unprecedented accuracy by space detectors such as PAMELA (Adriani et al. 2013, 2014), AMS-02 2 Aguilar et al. 2015a b), CREAM]3 (Yoon et al. 2017), DAMPE4 (An et al. 2019), and CALET5 (Maestro et al. 2020). Most of these direct measurements from Earth focus on the CR spectra above a few $\mathrm{GeV} /$ nuc, below which the fluxes are strongly affected by solar modulation. A few years ago, the Voyager 1 satellite passed through the termination shock and measured the lowenergy CR (LECR) spectra (from several MeV/nuc up to hun-dreds of MeV/nuc) beyond the Solar System (Cummings et al. 2016). The measurements outside the heliosphere are thought to be free of solar modulation and thus may represent the LECR spectra in the local interstellar medium. However, this information cannot be extrapolated to other parts of the Galaxy, in which the CRs are not homogeneously distributed (Aharonian et al. 2018; Jóhannesson et al. 2018; Baghmanyan et al. 2020). Fur-

\footnotetext{
1 A Payload for Antimatter Matter Exploration and Light-nuclei Astrophysics

2 The Alpha Magnetic Spectrometer

3 The Cosmic Ray Energetics and Mass experiment

4 The Dark Matter Particle Explorer

5 The CALorimetric Electron Telescope
}

thermore, the flux of LECRs can vary dramatically on a smaller scale because of higher energy losses and propagation effects, and the flux is usually much higher around possible CR acceleration sites. This is indirectly supported by studies of the interstellar ionization rates (in particular, see, e.g., Indriolo et al. 2009; Indriolo \& McCall 2012; Indriolo et al. 2015). These researches also argued for an LECR component in addition to the standard contribution by supernova remnants (SNRs), which is also supported by the observation of primary Be (Tatischeff \& Kiener 2011).

In this regard, $\gamma$-rays produced in interactions of CRs with the ambient gas can be used as a unique tool to study the spectral and spatial distributions of CRs throughout the Milky Way. For CRs whose kinetic energy exceeds the kinematic threshold of $\pi$-meson production, $E_{\mathrm{th}} \simeq 280 \mathrm{MeV} /$ nuc, the best $\gamma$ ray energy band for exploration is $0.1-100 \mathrm{GeV}$ because of the copious production of $\pi^{0}$-decay $\gamma$-rays and the potential of the currently most sensitive detector, Fermi LAT (Atwood et al. 2009). At energies below this kinematic threshold, the nuclear de-excitation lines provide the most straightforward information about the LECR protons and nuclei (e.g., Ramaty et al. 1979; Murphy et al. 2009).

In this paper, we treat the production of nuclear de-excitation lines by combining the recent advances in the modeling of nuclear reactions with the propagation and energy losses of subrelativistic and transrelativistic CRs. In Sec. 2 we calculate the distributions of LECRs near the sources, taking the processes of diffusion and energy losses into account. In Sec. 3 we describe the method for calculating the emissivity of de-excitation $\gamma$-ray lines using the code TALYS (Koning et al. 2008). We then present the 
results and discuss their implications. Finally, we summarize the main results in Sec. 4

\section{Propagation of LECR protons}

The energy losses of CR protons with kinetic energies above $\sim 1 \mathrm{GeV}$ are dominated by inelastic nuclear reactions (see Fig 1), and the corresponding cooling weakly depends on energy. As a result, the propagation has very little effect on the initially injected CR spectral shape. At lower energies, the losses are dominated by the ionization and heating of the ambient medium. In this regime, as shown in Fig 1 the cooling time is energy dependent, thus the propagation can significantly distort the initial spectrum. On the other hand, because of the energy-dependent particle diffusion, LECRs propagate more slowly than relativistic CRs. Consequently, the spatial distribution of LECRs should be much more inhomogeneous than that of high-energy CRs. LECRs are expected to be concentrated in the vicinity of their accelerators with energy distributions that strongly depend on the distance to their production sites. The propagation of LECRs in the proximity of their sources should therefore be treated with great care and depth.

As introduced in Padovani et al. (2009), $L=-d E / d N_{\mathrm{H}_{2}}$, represents the energy loss-rate per column density $d N_{\mathrm{H}_{2}}$, then the ionization cooling time for protons colliding with a medium atomic hydrogen density $n$ can be estimated by $\tau \sim \frac{E}{\operatorname{Ln} \beta c}$, where $\beta=v / c$. For protons with kinetic energy $10 \mathrm{MeV}$ that collide with $\mathrm{H}_{2}, L \simeq 2 \times 10^{-16} \mathrm{eV} \mathrm{cm}^{2}$ (see Fig.7 in Padovani et al.2009). The cooling time of $10 \mathrm{MeV}$ protons in a dense environment with $n \geq 100 \mathrm{~cm}^{-3}$ therefore is about several thousand years, much shorter than the duration of typical CR accelerators such as SNRs. Therefore we adopt the steady-state solution for continuous injection to estimate the spectra of LECRs. To be more specific, we assume a continuous injection of protons from a stationary point source. Then the steady-state energy and radial distribution distribution of the LECR protons is obtained by application of the analytical solution derived by Atoyan et al. (1995),

$F_{\mathrm{p}}(r, E)=\frac{1}{8 \pi^{3 / 2} P(E)} \int_{E}^{\infty} \frac{Q(x)}{[\Delta u(E, x)]^{3 / 2}} \times \exp \left(-\frac{r^{2}}{4 \Delta u(E, x)}\right) \mathrm{d} x$.

Here $Q(E)$ represents the $\mathrm{CR}$ injection rate, $P(E)$ is the energy loss-rate of CR protons summarized in Padovani et al. (2009), $r$ represents the radial distance to the source, and $\Delta u(E, x)=$ $\int_{E}^{x} \frac{D\left(E^{\prime}\right)}{P\left(E^{\prime}\right)} d E^{\prime}$, where $D(E)$ is the energy-dependent diffusion coefficient of CRs. In general, this is a quite uncertain parameter that depends on the level of turbulence in the environment. In the Galaxy, it is derived from observations of secondary CRs. We adopted the diffusion coefficient derived for Galactic CRs at energies above $1 \mathrm{GeV}$ (Strong et al. 2007) and assumed that it can be extrapolated to low energies down to $1 \mathrm{MeV}$ : $D(E)=4 \times 10^{28} \chi(E / 1 \mathrm{GeV})^{0.5} \mathrm{~cm}^{2} \mathrm{~s}^{-1}$. Because the turbulence level near the CR sources is high, the diffusion could be much slower (Malkov et al. 2011; D’Angelo et al. 2018). Effects like this have previously been observed in the $\gamma$-ray band. The electron halos near pulsars reveal a small diffusion coefficient Abeysekara et al. 2017; Di Mauro et al. 2019). To take this effect into account, we considered a broad range of the parameter $\chi=1,0.1,0.01$. Here the total proton injection rate $Q$, integrated from $1 \mathrm{MeV}$ to $100 \mathrm{MeV}$, and the distance of the source $d$, are fixed by the value of parameter $Q / d^{2}=10^{38} \mathrm{erg} \mathrm{s}^{-1} \mathrm{kpc}^{-2}$.

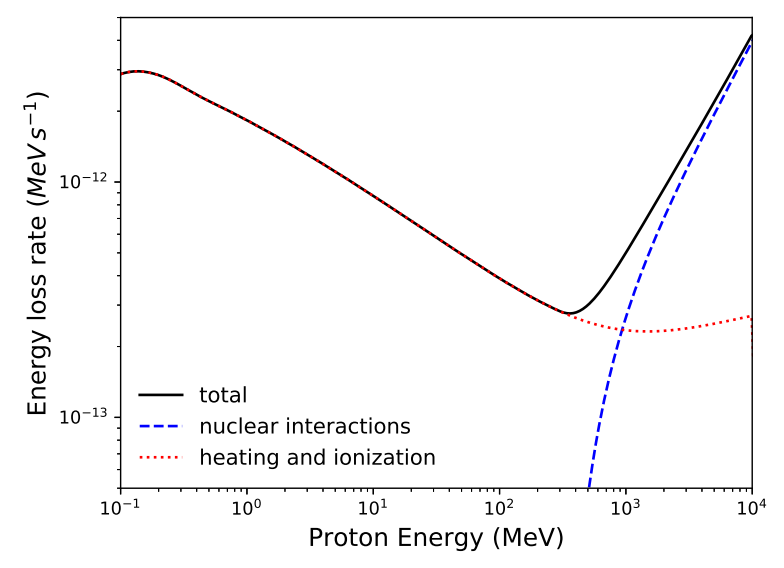

Fig. 1: Energy loss-rate as a function of kinetic energy for protons colliding with atomic hydrogen. The number density of atomic hydrogen $n$ is assumed to be $1 \mathrm{~cm}^{-3}$. The dashed blue line indicates the loss rate due to p-p inelastic interactions, and the dotted red line represents the energy losses caused by heating and ionization.

The injection spectrum of CRs depends on the acceleration mechanism and the conditions inside the accelerator such as the turbulence level and the magnetic field. Typically (although not always), in the case of diffusive shock acceleration, for instance, the distribution of accelerated particles can be presented as a power law in momentum $p, Q(p)=Q_{0} p^{-s}$ (see, e.g., Amato 2014). In this paper, it is more convenient to write the distribution in terms of kinetic energy $E, Q(E)=Q_{0} p^{-s} / \beta$, where $Q_{0}$ is the normalization parameter derived from the power of the $C R$ source. By solving Eq11 we obtain the steady-state distribution of protons at different radial distances $r$ to the source.

The energy spectra of CR protons diffusing from the site of their acceleration are shown in Fig 2 In the left panel of Fig 2 the curves are calculated for four injection spectra of protons with power-law index $s=1.0,2.0,3.0$, and 4.0, in which the radial distance $r=10 \mathrm{pc}$, the medium density $n=1 \mathrm{~cm}^{-3}$, and the parameter $\chi=1$. In the right panel of Fig 2, we present the $\mathrm{CR}$ spectra for the injection spectrum with $s=2.0$ at various distances, in which $r=1 \mathrm{pc}, 10 \mathrm{pc}$, and $100 \mathrm{pc}$. By changing the diffusion coefficient, that is, the parameter $\chi=1,0.1$, and 0.01 , we find that for a small diffusion coefficient, the energy spectra of protons become very hard, especially at large distances from the source.

In Fig. 3 we show the radial dependence of proton fluxes. In the left panel, we show the effect of the diffusion coefficient on the flux of $10 \mathrm{MeV}$ protons. At small distances from the source, their proton flux is significantly higher for slow diffusion, but at large distances, the flux drops as the cooling becomes an important factor. The contrast of the spectra under different assumptions of $n$ (the red and black lines in the left panel of Fig 3 also shows the effect of the ambient gas density on the CR spectrum. As expected, because of enhanced energy losses, the radial distribution of the CR fluxes become sharper than $1 / r$ at larger distances, $r \geq 10 \mathrm{pc}$. In the right panel, we show the $r$-profiles for different $\mathrm{CR}$ energies calculated for the parameter $\chi=1$. For the radial profile of the $\mathrm{CR}$, the flux at $1 \mathrm{GeV}$ is close to $1 / r$. This is consistent with predictions for the continuous injection in Atoyan et al. (1995), as long as the energy losses are negligible. At low energies, $E \leq 1 \mathrm{GeV}$ because of the high energy losses 
and slow propagation, we see a stronger radial dependence of the CR flux. Namely, at small distances, the flux follows the $1 / r$ profile, but at larger distances the cooling starts to play significant role and causes the radial distribution to become as steep as $1 / r^{3}$. For $1 \mathrm{MeV}$ protons, the transition between $1 / r$ and $1 / r^{3}$ takes place at just several pc. Last but not least, we note that the hardening of the spectrum of CR protons at low energies in the local interstellar medium, as shown by the gray line in Fig. 2 , fitted by Phan et al. (2018) using data from Voyager 1 and AMS02 detectors), can be naturally explained by the propagation and energy losses.

In the derivation of Eq.(1), only the diffusion and energy loss of protons are taken into account. Meanwhile, the advection may also play a non-negligible role in the vicinity of CR sources. In this case, an advection term $V \frac{\partial F_{\mathrm{p}}(r, E)}{\partial r}$ should be added to the propagation equation, where $V$ is the advection velocity. By dimensional analysis, the advection dominates diffusion in the region $r>D / V \sim 30 \mathrm{pc} \frac{D}{10^{27} \mathrm{~cm}^{2} \mathrm{~s}^{-1}} / \frac{V}{100 \mathrm{~km} / \mathrm{s}}$. The Alfvénic velocity in the interstellar medium is estimated as several $\mathrm{km} / \mathrm{s}$ assuming the density of $\sim 1 \mathrm{~cm}^{-3}$ and magnetic fields of about $3 \mu \mathrm{G}$ (see, e.g., Han 2017). In this case the advection can be neglected close to the source. However, for a strong outflow of gas or a stellar wind near the source, with a speed as as high as $1000 \mathrm{~km} / \mathrm{s}$ (see, e.g., Domingo-Santamaría \& Torres 2006), at distances $r \geq 0.1 \mathrm{pc}$, the advection can dominate the diffusion, resulting in the steadystate solution

$F_{\mathrm{p}}(r, E)=\frac{Q\left(E_{0}\right)}{4 \pi r^{2} \beta c}$,

where $E_{0}$ is obtained from the equation $\frac{r}{V}=\int_{E}^{E_{0}} \frac{d E^{\prime}}{P\left(E^{\prime}\right)}$. The corresponding CR fluxes are shown in Fig 4 . The fast advection results in the strong suppression of CR flux even compared with the fast diffusion case $(\chi=1)$ in the vicinity of the source.

Moreover, in the calculation above we assumed that the power-law diffusion coefficient extends to low energy. Recent analyses show indications of a low-energy break in the diffusion coefficient in the ISM (Vittino et al. 2019; Weinrich et al. 2020), which was also predicted in Ptuskin (2006) because the damping of CRs terminates the cascade of turbulence and induces faster diffusion for LECRs. However, it is not straightforward to adopt this scenario in the vicinity of the CR sources, where the external turbulence is also stronger by an order of magnitude. The detailed calculation requires a self-consistent treatment of the CR propagation and magnetic turbulence development and damping near the CR sources, which need further investigations.

\section{Rates and spectral features of the de-excitation $\gamma$-ray line emission}

\subsection{Method for estimating the $\gamma$-ray line emission}

The interactions of LECRs with the surrounding gas excite nuclei that belong to LECRs and to the ambient medium. The almost prompt de-excitation of these nuclei leads to the $\mathrm{MeV} \gamma$ ray line emission. The main production of $\gamma$-ray lines proceeds through (1) energetic protons and $\alpha$-particles as projectiles interacting with heavier elements of the ambient gas, that is, direct reactions, and (2) interactions of heavy nuclei of LECRs with the hydrogen and helium of the ambient gas, that is, inverse reactions. Both channels were taken into account in the calculations of the $\gamma$-ray emission. For simplicity, we only considered the most abundant stable isotope of elements, including $\mathrm{C}, \mathrm{N}, \mathrm{O}$, $\mathrm{Ne}, \mathrm{Na}, \mathrm{Mg}, \mathrm{Al}, \mathrm{Si}, \mathrm{S}, \mathrm{Ca}, \mathrm{Ar}, \mathrm{Fe}$, and $\mathrm{Ni}$, and disregarded the isotopes with lower abundance, such as ${ }^{3} \mathrm{He},{ }^{13} \mathrm{C}$, and ${ }^{22} \mathrm{Ne}$. The abundance of these element species in the LECRs were extracted from Table 3 of Cummings et al. (2016). For the composition of the ambient medium, we used the recommended present-day solar abundances of Lodders (2010). These data are compiled in Tab.1. Moreover, we assumed that the injected energy spectra of energetic $\alpha$ and other species have the same shape as that of CR protons, $F_{i}(r, E) \propto F_{\mathrm{p}}(r, E)$, where $E$ is expressed as kinetic energy per nucleon, and we calculated their propagated spectra using the same method as described in Sec 2 . The energy lossrate of heavy nuclei can be significantly different from that of protons. To account for these differences, the energy loss-rate for each nucleus species including ionization and nuclear interaction were derived from formulae in Mannheim \& Schlickeiser (1994) and cross sections in Sihver et al. (1993).

To calculate emissivities of the de-excitation $\gamma$-ray line lines, we used the code TALYS (version 1.95) 6, which is a flexible and user-friendly program aimed at a complete and accurate simulation of nuclear reactions (Koning et al. 2008). The newly updated version of TALYS provides adequate precision of cross sections at projectile energies $<1 \mathrm{GeV}$ Koning et al. 2014). For a better match with the experiment data, we modified the deformation files of ${ }^{14} \mathrm{~N},{ }^{20} \mathrm{Ne}$, and ${ }^{28} \mathrm{Si}$ using the results of Benhabiles-Mezhoud et al. (2011). Then, following the approach of Murphy et al. (2009), we divided the $\gamma$-ray emission into three categories: the explicit lines, the quasi-continuum consisting of discrete lines, and the continuum. All the discrete lines with the TALYS-calculated cross sections with $>10$ mbarn were selected as explicit lines. Those with smaller cross sections were treated as quasi-continuum. The continuum component produced by the so-called direct, pre-equilibrium, and compound reactions (see the TALYS user manual for a detailed description) was considered as well. Because in the updated version of TALYS the projectile energy relevant to reactions with involvement of $\alpha$-particles is limited to $250 \mathrm{MeV} /$ nuc, we extrapolated these line production cross sections to $1 \mathrm{GeV} /$ nuc and assumed that the continuum production cross section remains constant when the projectile energies exceed $250 \mathrm{MeV} /$ nuc. The production cross sections of the specific lines listed in the compilation of Murphy et al. (2009) were extracted from Table 3 therein. For each explicit line, we derived the line profile according to the method proposed in Ramaty et al. (1979).

\subsection{Results and discussion}

For the given compositions of LECRs and the ambient gas, the detectability of the $\gamma$-ray lines, that is, their flux integrated over the entire region occupied by LECRs, depends on several parameters. First of all, the LECR injection rate of source $Q$ versus its distance $d$, which could be represented by the parameter $Q / d^{2}$, the density of the ambient gas $n$, the speed of propagation of LECRs, and their original spectral shape. With the method described above, we first calculated the emissivity of the $\gamma$-ray line emission at different radial distances $r$ to a hypothetical CR source that continuously injects CRs with a power-law index $s=2$ and $Q / d^{2}=10^{38} \mathrm{erg} \mathrm{s}^{-1} \mathrm{kpc}^{-2}$ into the surrounding medium of $n=100 \mathrm{~cm}^{-3}$. The results, as shown in Fig 5, show a significant effect of the propagation process on the emissivities of the $\gamma$-ray line emission. We then integrated the intensity of several representative narrow lines along the line of sight at different angular distances $\theta$, assuming the distance of the source $d$ is $1 \mathrm{kpc}$. As shown in Fig 6 the fluxes drop sharply with $\theta$

${ }^{6}$ https://tendl.web.psi.ch/tendl_2019/talys.html 

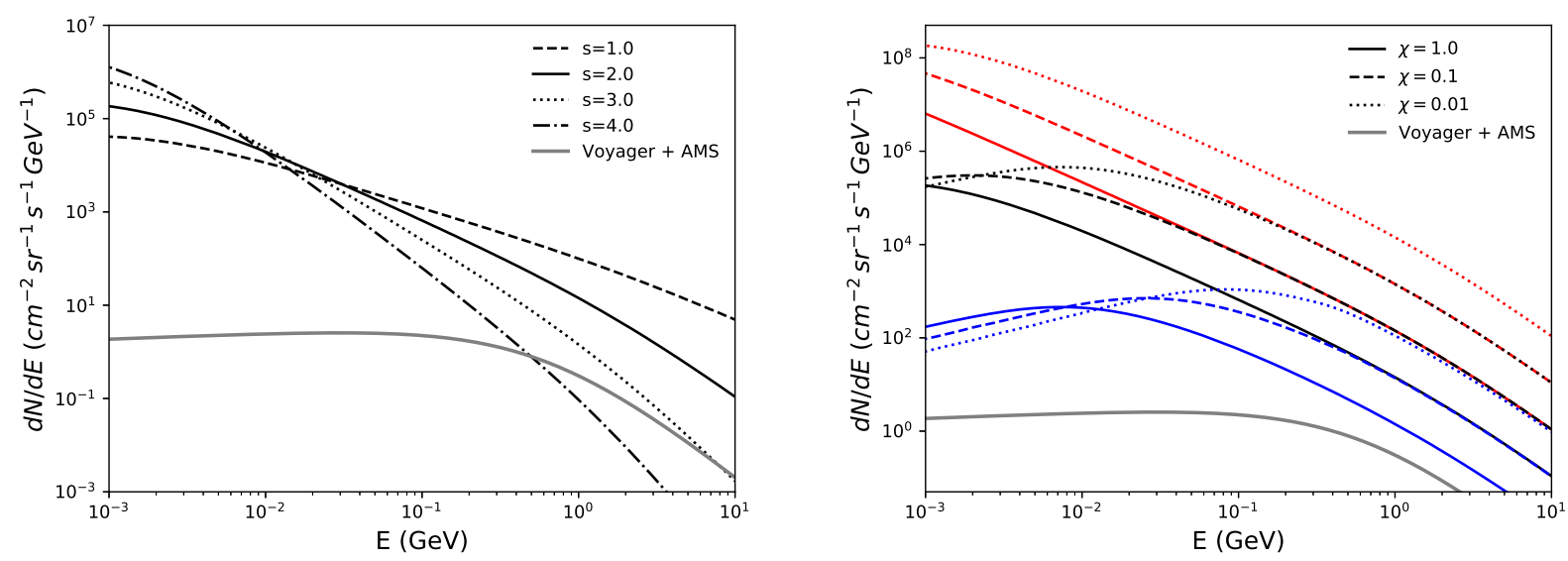

Fig. 2: Calculated proton spectra under different assumptions. Left panel: Proton spectra at the radical distance $r=10 \mathrm{pc}$ from the hypothetical CR source with various injection spectral indices, in which $s=1.0$ (dashed line), 2.0 (solid line), 3.0 (dotted line), and 4.0 (dash-dotted line), assuming a diffusion coefficient parameter $\chi=1.0$. Right panel: Proton spectra with injection spectral index $s=2.0$ under various assumptions of diffusion coefficients (solid lines for $\chi=1.0$, dashed lines for $\chi=0.1$, and dotted lines for $\chi=$ 0.01 ) at different radial distances, where $r=1 \mathrm{pc}$ (red), $10 \mathrm{pc}$ (black), or $100 \mathrm{pc}$ (blue). In both panels, $Q / d^{2}=1 \times 10^{38} \mathrm{erg} \mathrm{s}^{-1} \mathrm{kpc}^{-2}$ and $n=1 \mathrm{~cm}^{-3}$ are assumed, and the gray lines represents the fit of the CR proton intensity from Phan et al. (2018) based on the measured fluxes of local CRs reported by Voyager 1 (Cummings et al.|2016) and AMS-02 (Aguilar et al.|2015a).
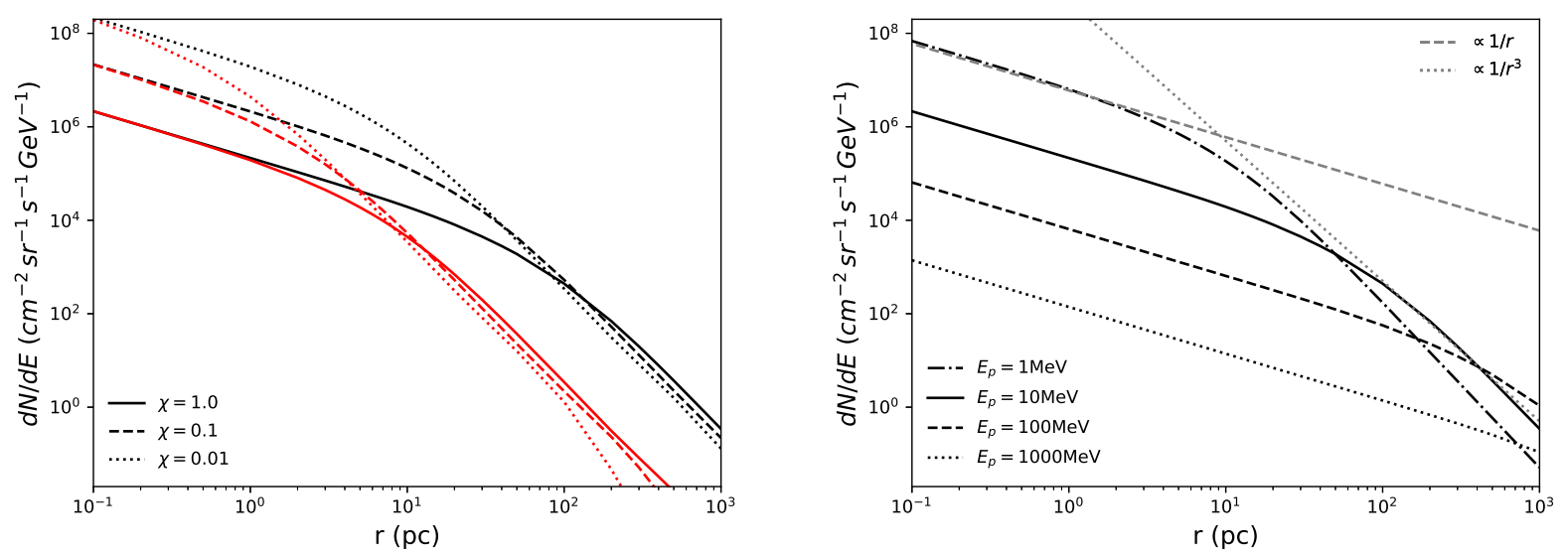

Fig. 3: Radial distribution of protons with certain kinetic energies. Left panel: Flux of $10 \mathrm{MeV}$ protons as a function of radial distance $r$ under different assumptions of medium density (black lines for $n=1 \mathrm{~cm}^{-3}$, and red lines for $n=100 \mathrm{~cm}^{-3}$ ) and diffusion coefficient (solid lines for $\chi=1.0$, dashed lines for $\chi=0.1$, dotted lines for $\chi=0.01$ ). Right panel: Flux of protons with different kinetic energies $E_{p}$ as a function of radial distance $r$, assuming $n=1 \mathrm{~cm}^{-3}$, and $\chi=1$. For comparison, two radial profiles $1 / r$ (dashed gray line) and $1 / r^{3}$ (dotted gray line) are also shown. In both panels, we assume $s=2.0$ and $Q / d^{2}=1 \times 10^{38} \mathrm{erg} \mathrm{s}^{-1} \mathrm{kpc}^{-2}$.

and become almost negligible at $\theta \sim 1.5^{\circ}$, which corresponds to a radial distance $r \sim 25 \mathrm{pc}$. Unfortunately, the angular resolution of $\mathrm{MeV} \gamma$-ray detectors, including those planned for the foreseeable future, such as e-Astrogam (de Angelis et al. 2018) and AMEGO (McEnery et al. 2019), is quite limited, about $1.5^{\circ}$ , thus the angular distribution of these lines can be detected only for very nearby objects, $d \ll 1 \mathrm{kpc}$. In the following discussion we therefore treat the $\gamma$-ray emission around the injection point as a point-like source, and calculate the differential flux of this $\gamma$-ray source by integrating the $\gamma$-ray emission within $25 \mathrm{pc}$ from the injection site,

$F_{\gamma}\left(E_{\gamma}\right)=\frac{1}{4 \pi d^{2}} \int_{0}^{r_{25}} 4 \pi r^{2} I\left(E_{\gamma}, r\right) \mathrm{d} r$,

where $I\left(E_{\gamma}, r\right)$ is the $\gamma$-ray emissivity at the distance $r$. In the left panel of Fig. 7, we show the differential fluxes of $\gamma$-rays calculated for $s=2.0, Q / d^{2}=10^{38} \mathrm{erg} \mathrm{s}^{-1} \mathrm{kpc}^{-2}, n=1 \mathrm{~cm}^{-3}$, and $\chi=1$. The brightest lines are $0.847,1.634,4.44$, and 6.13 $\mathrm{MeV}$ lines resulting from the de-excitation of ${ }^{56} \mathrm{Fe},{ }^{20} \mathrm{Ne},{ }^{12} \mathrm{C}$, and ${ }^{16} \mathrm{O}$, respectively, from their first or second excited state to ground state. The direct (narrow) and inverse (broad) components linked to the nuclei of LECRS and the ambient gas both contribute substantially to the total flux. The direct processes contribute to the narrow line structures, while the inverse processes are responsible for the broad lines and thus contribute mostly to the continuum emissions. In the right panel of Fig. 7 we compare the fluxes of $\gamma$-ray emission integrated within $25 \mathrm{pc}$ radial distance from the source for two different gas densities $n=1 \mathrm{~cm}^{-3}$ and $n=100 \mathrm{~cm}^{-3}$ and different diffusion coefficient parameters $(\chi=1$ and 0.01$)$. A denser environment and lower 


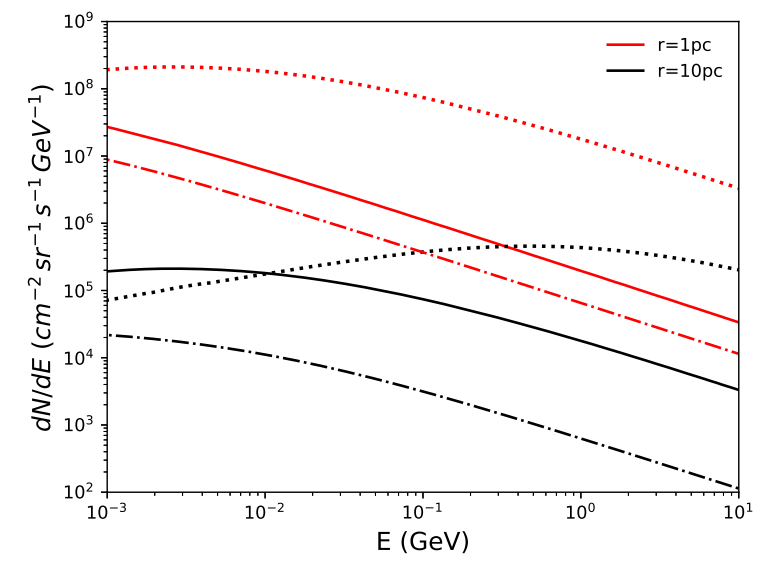

Fig. 4: Proton spectra at different radial distances, in which $r=$ $1 \mathrm{pc}$ (red) or $10 \mathrm{pc}$ (black). Assuming $s=2.0, n=1 \mathrm{~cm}^{-3}$, and $Q / d^{2}=1 \times 10^{38} \mathrm{erg} \mathrm{s}^{-1} \mathrm{kpc}^{-2}$, the solid $(\chi=1.0)$ and dotted lines $(\chi=0.01)$ are calculated for the diffusion using Eq.11, and the dash-dotted lines are calculated for advection using Eq. (2) for $V=1000 \mathrm{~km} / \mathrm{s}$.

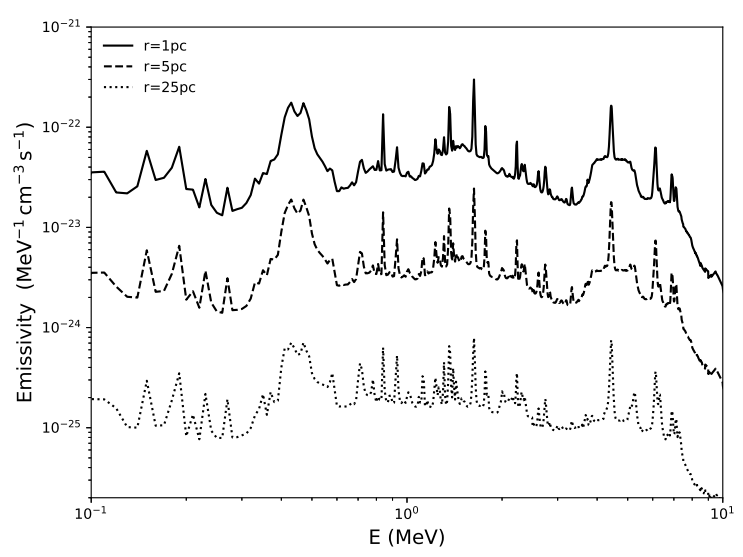

Fig. 5: Calculated $\gamma$-ray emissivity at different radial distances from the hypothetical CR source, in which $r=1 \mathrm{pc}$ (solid line), $5 \mathrm{pc}$ (dashed line), and $25 \mathrm{pc}$ (dotted line), assuming $Q / d^{2}=1 \times$ $10^{38} \mathrm{erg} \mathrm{s}^{-1} \mathrm{kpc}^{-2}, d=1 \mathrm{kpc}, s=2.0$, and $n=100 \mathrm{~cm}^{-3}$.

diffusion coefficient can enhance the flux of $\gamma$-ray line emission. The integrated fluxes of the narrow 4.44 MeV line emission, calculated for different combinations of $n$ and $\chi$, are presented in Table 2. The $4.44 \mathrm{MeV} \gamma$-ray line flux sensitivity of the proposed telescopes e-ASTROGAM (de Angelis et al. 2018) and AMEGO (McEnery et al. 2019) is $\sim 10^{-7} \mathrm{ph} \mathrm{cm}^{-2} \mathrm{~s}^{-1}$ for the observation time $T_{\mathrm{obs}}=1 \mathrm{yr}$. The comparison with Table 2 shows that in very dense environments with $n \geq 100 \mathrm{~cm}^{-3}$ and slow particle diffusion, the LECR sources can be revealed through $\gamma$ ray lines provided that the parameter $Q / d^{2}$ is not significantly smaller than $10^{38} \mathrm{erg} \mathrm{s}^{-1} \mathrm{kpc}^{-2}$. In all figures above, the composition of the ambient medium is fixed to the solar abundance. However, in certain astronomical locations, the composition might be different, in particular, it might be enhanced by heavy elements in metal-rich environments, such as the Galactic center (Benhabiles-Mezhoud et al. 2013), or in the young SNR Cas A (Summa et al. 2011). The higher abundance can dramatically en-

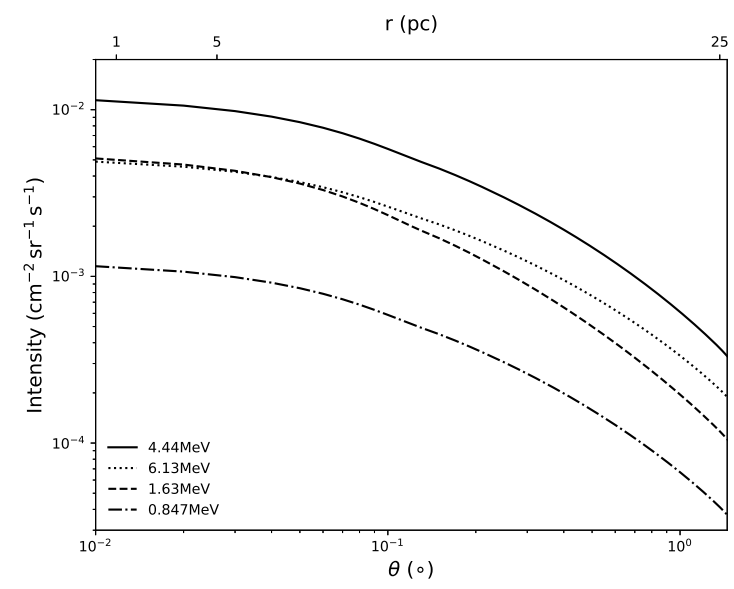

Fig. 6: Integrated flux of strong narrow lines at 4.44 MeV (solid line), $6.13 \mathrm{MeV}$ (dotted line), 1.63 MeV (dashed line), and 0.847 $\mathrm{MeV}$ (dash-dotted line), as a function of angular distance $\theta$ (or radial distance $r$ ) from the hypothetical CR source. $Q / d^{2}=1 \times$ $10^{38} \mathrm{erg} \mathrm{s}^{-1} \mathrm{kpc}^{-2}, d=1 \mathrm{kpc}, s=2.0$, and $n=100 \mathrm{~cm}^{-3}$ are assumed for the calculation.

hance the $\gamma$-ray line fluxes and makes these sources prime targets for observations with next-generation $\mathrm{MeV} \gamma$-ray detectors.

So far, we did not include in calculations the $\gamma$-ray channel linked to $p p$ interactions with the production and decay of $\pi^{0}$ mesons. The relative contribution of this channel strongly depends on the proton spectrum, especially on the continuation of the proton spectrum beyond the kinematic threshold of $\pi$ meson production. Setting $Q / d^{2}=1 \times 10^{38} \mathrm{erg} \mathrm{s}^{-1} \mathrm{kpc}^{-2}$ and $n=1 \mathrm{~cm}^{-3}$, in Fig 8 we show the differential spectra of $\gamma$ radiation consisting of the nuclear de-excitation lines and the $\pi^{0}$ decay $\gamma$-rays integrated within the region of radius $25 \mathrm{pc}$ around the source, assuming that the initial proton spectrum has a simple power-law distribution with index $s=2$ without cutoff (the left panel) or with an exponential cutoff at $E_{\text {cut }}=100 \mathrm{MeV}, 300 \mathrm{MeV}$, and $1 \mathrm{GeV}$ (the right panel). The left panel of Fig 8 shows that $\pi^{0}$-decay $\gamma$-rays exceed the luminosity of nuclear lines. The reason is that the energy losses cause the spectrum of CRs below $100 \mathrm{MeV}$ to become very hard, thus the production rate of nuclear lines is suppressed. On the other hand, the emissivity of $\pi^{0}$-decay $\gamma$-rays is dramatically suppressed when the cutoff in the spectrum of LECRs is close to the threshold of $\pi^{0}$-decay production around $280 \mathrm{MeV}$, as demonstrated in the right panel of Fig 8

It is also interesting to compare our results with those in Benhabiles-Mezhoud et al. (2013), which were calculated for the inner Galaxy. The results in Benhabiles-Mezhoud et al. (2013) reveal a significantly sharper profile than our results. The differences come mainly from the twice solar abundance used in their calculation, and thus more contribution from the direct processes. Benhabiles-Mezhoud et al. (2013) moreover calculated a diffuse emission in a much larger region, in which the variation in the CR spectrum on a small scale may have only a minor effect on the final results. The limited angular resolution of the current and planned $\mathrm{MeV}$ instruments means that such diffuse emission may be a better target than point sources. On the other hand, our calculations treated the spectral and density variation of CRs carefully and were aimed at the nearby CR sources. 

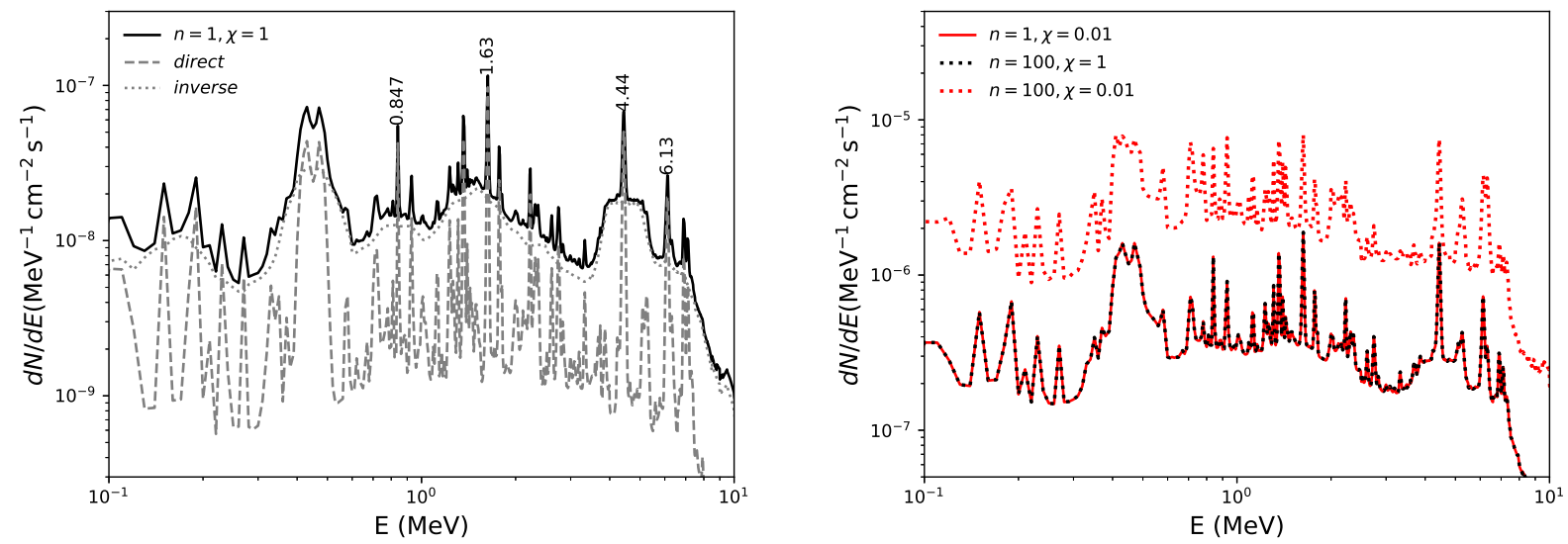

Fig. 7: Comparison of the integrated $\gamma$-ray differential fluxes for different $n\left(1 \mathrm{~cm}^{-3}\right.$ or $\left.100 \mathrm{~cm}^{-3}\right)$ and $\chi(1.0$ or 0.01$)$. In the left panel, the solid line show the total $\gamma$-ray flux integrated within $r=25 \mathrm{pc}$ from the hypothetical CR source, assuming $\chi=1.0$ and $n=1 \mathrm{~cm}^{-3}$, and the dashed and dotted lines correspond to the contributions from the direct and inverse processes, respectively. In the right panel, the lines represent the total integrated $\gamma$-ray line emission under different assumptions of $n$ and $\chi$. In both panels, we assume $Q / d^{2}=1 \times 10^{38} \mathrm{erg} \mathrm{s}^{-1} \mathrm{kpc}^{-2}, s=2.0$.
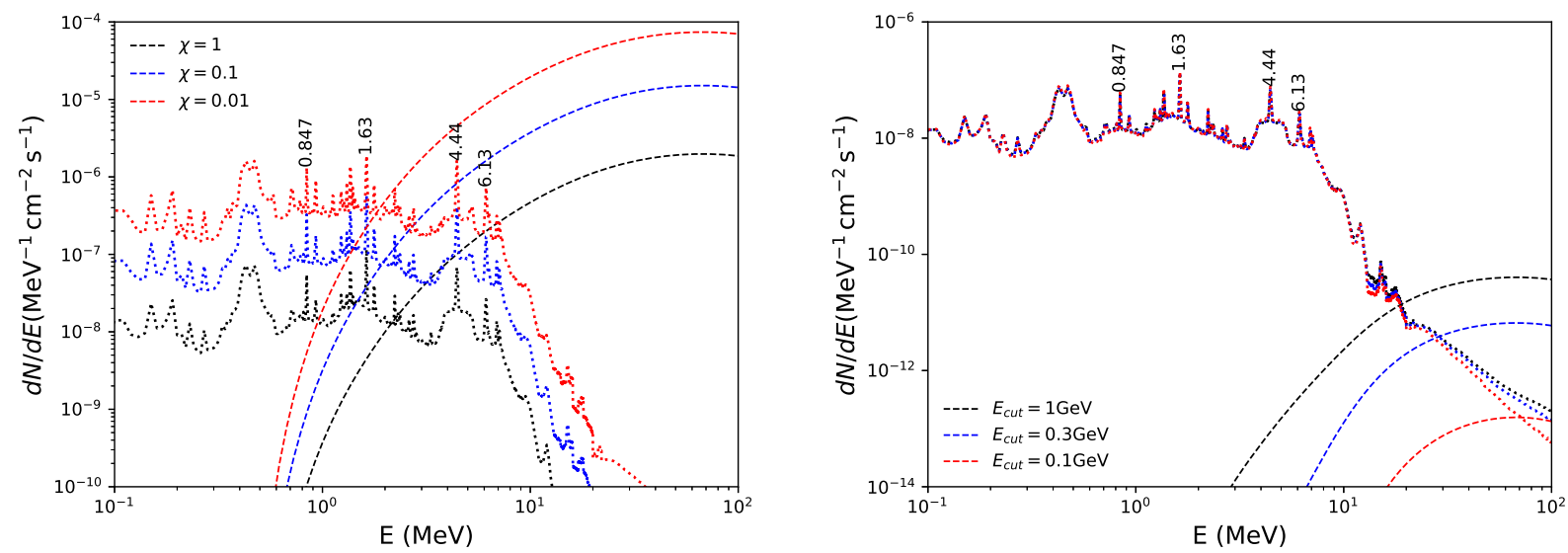

Fig. 8: Comparison of $\gamma$-ray fluxes resulting from de-excitation of nuclei (dotted lines) and $\pi^{0}$-decay process (dashed lines) integrated within $25 \mathrm{pc}$ from the hypothetical CR source. In the left panel, we assume a proton spectrum $F_{\mathrm{p}}(E) \propto E^{-2}$ with various diffusion coefficient parameters, in which $\chi=1.0$ (black), 0.1 (blue), or 0.01 (red). In the right panel, we assume $\chi=1.0$ and proton spectra $F_{\mathrm{p}}(E) \propto E^{-2} \exp \left(-E / E_{\text {cut }}\right)$ with various cutoff energies, in which $E_{\text {cut }}=1 \mathrm{GeV}$ (black), $300 \mathrm{MeV}$ (blue), or $100 \mathrm{MeV}$ (red). Meanwhile, $Q / d^{2}=10^{38} \mathrm{erg} \mathrm{s}^{-1} \mathrm{kpc}^{-2}$ and $n=1 \mathrm{~cm}^{-3}$ are assumed in both panels.

\section{Summary}

Together with magnetic fields and turbulent gas motions, Galactic CRs play a dominant role in the energy balance of the interstellar medium. Although subrelativistic (suprathermal) particles supply a substantial fraction of the CR pressure, the real contribution of LECRs remains uncertain. Because of the slow propagation and severe energy losses, the local LECRs make negligible contributions to the fluxes beyond $100 \mathrm{pc}$. For the same reason, LECRs are expected to be inhomogeneously distributed in the Galactic disk; subrelativistic protons and nuclei are expected to be concentrated around their acceleration sites. Because all potential CR source populations (SNRs, stellar clusters, individual stars, etc.) are linked in one way or another to the star-forming region, the effective confinement of LECRs in these regions is expected to produce feedback that stimulates the star formation through the ionization of the nearby molecular clouds. LECRs play a significant role also in the chemistry of the interstellar medium. Thus an unbiased, observation based information about LECRs at the sites of their concentrations in the Milky Way is crucial for understanding the fundamental processes linked to the dynamics and chemistry of the interstellar medium, star formation, etc. The most direct channel of information is provided by nuclear de-excitation $\gamma$-ray lines resulting from the interactions of protons and nuclei of LECRs with the ambient gas.

We explored the effect of the initial spectra shape, energy loss, and diffusion coefficient on the spatial and energy distributions of LECRs around the source that continuously injects accelerated protons and nuclei into the surrounding medium. LECRs, lose much energy during propagation, especially at energies below $100 \mathrm{MeV} /$ nuc. At large distances, depending on the diffusion coefficient, their radial profiles become steeper than $1 / r$ (see Fig 3), which is the typical radial distribution of particles at higher energies at which the energy losses can be neglected. We 
Table 1: Elemental composition

\begin{tabular}{lccc}
\hline $\mathrm{Z}$ & Element & Local CR $^{a}$ & Solar $^{b}$ \\
\hline 1 & $\mathrm{H}$ & 1 & 1 \\
2 & $\mathrm{He}$ & $8.140 \times 10^{-2}$ & $8.414 \times 10^{-2}$ \\
6 & $\mathrm{C}$ & $1.671 \times 10^{-3}$ & $2.455 \times 10^{-4}$ \\
7 & $\mathrm{~N}$ & $2.444 \times 10^{-4}$ & $7.244 \times 10^{-5}$ \\
8 & $\mathrm{O}$ & $1.570 \times 10^{-3}$ & $5.370 \times 10^{-4}$ \\
10 & $\mathrm{Ne}$ & $1.507 \times 10^{-4}$ & $1.122 \times 10^{-4}$ \\
11 & $\mathrm{Na}$ & $1.784 \times 10^{-5}$ & $1.950 \times 10^{-6}$ \\
12 & $\mathrm{Mg}$ & $2.264 \times 10^{-4}$ & $3.467 \times 10^{-5}$ \\
13 & $\mathrm{Al}$ & $3.302 \times 10^{-5}$ & $2.884 \times 10^{-6}$ \\
14 & $\mathrm{Si}$ & $1.898 \times 10^{-4}$ & $3.388 \times 10^{-5}$ \\
16 & $\mathrm{~S}$ & $2.087 \times 10^{-5}$ & $1.445 \times 10^{-5}$ \\
18 & $\mathrm{Ar}$ & $4.554 \times 10^{-6}$ & $3.162 \times 10^{-6}$ \\
20 & $\mathrm{Ca}$ & $1.195 \times 10^{-5}$ & $2.042 \times 10^{-6}$ \\
26 & $\mathrm{Fe}$ & $1.152 \times 10^{-4}$ & $2.884 \times 10^{-5}$ \\
28 & $\mathrm{Ni}$ & $6.452 \times 10^{-6}$ & $1.660 \times 10^{-6}$ \\
\hline
\end{tabular}

Notes. ${ }^{(a)}$ The LECR abundance according to the Voyager 1 measurement (see Cummings et al. 2016, Table 3). ${ }^{(b)}$ The recommended present-day solar abundances extracted from the Table 6 of Lodders (2010)

Table 2: Integrated 4.44 MeV line flux under different assumptions of $n$ and $\chi$

\begin{tabular}{cc|c}
\hline $\begin{array}{c}n \\
\left(\mathrm{~cm}^{-3}\right)\end{array}$ & $\chi$ & $\begin{array}{c}\text { Flux } \\
\left(\text { photon cm } \mathrm{cm}^{-2} \mathrm{~s}^{-1}\right)\end{array}$ \\
\hline 1 & 1.0 & $5.49 \times 10^{-9}$ \\
1 & 0.1 & $3.25 \times 10^{-8}$ \\
1 & 0.01 & $1.25 \times 10^{-7}$ \\
100 & 1.0 & $1.25 \times 10^{-7}$ \\
100 & 0.1 & $3.16 \times 10^{-7}$ \\
100 & 0.01 & $5.96 \times 10^{-7}$ \\
\hline
\end{tabular}

Notes. The injection rate of protons $Q / d^{2}=10^{38} \mathrm{erg} \mathrm{s}^{-1} \mathrm{kpc}^{-2}$ and spectral index $s=2.0$ are assumed. The FWHM width of $4.44 \mathrm{MeV}$ narrow line $\Delta E$ is $\sim 100 \mathrm{keV}$. Details is described in Sec 3.2

calculated that characteristics of the nuclear $\gamma$-ray line emission initiated by interactions of LECRs with the ambient gas using the cross sections gathered from experimental data and theoretical modeling with the code TALYS-1.95. We found that for the standard diffusion coefficient characterizing the propagation of CRs in the interstellar medium, the $\gamma$-ray emission is mainly produced in regions around the source within $\sim 25 \mathrm{pc}$. The slower diffusion with the parameter $\chi<1$ makes the $\gamma$-ray source more compact and brighter. The expected $\gamma$-ray fluxes are unfortunately well below the sensitivity of current $\gamma$-ray detectors, however. With the arrival of the proposed $\gamma$-ray detectors that are dedicated to low energies, such as e-ASTROGAM (de Angelis et al. 2018) and AEMGO (McEnery et al. 2019), the detection of nuclear $\gamma$ rays, in particular, the lines at $0.847,1.63,4.44$, and $6.13 \mathrm{MeV}$ of nearby (within a few kpc) accelerators of LECRs would become feasible provided that the LECR accelerators are surrounded by dense $\left(\geq 100 \mathrm{~cm}^{-3}\right)$ gaseous regions in which LECRs propagate significantly more slowly than in the interstellar medium, $\chi \ll 1$.

Acknowledgements. Bing Liu thanks Jurgen Kiener for providing very helpful information on the modification of TALYS structure files, and is supported by the Fundamental Research Funds for the Central Universities. Ruizhi Yang is supported by the NSFC under grants 11421303 and the national youth thousand talents program in China.

\section{References}

Abeysekara, A. U., Albert, A., Alfaro, R., et al. 2017, Science, 358, 911 Adriani, O., Barbarino, G. C., Bazilevskaya, G. A., et al. 2014, ApJ, 791, 93 Adriani, O., Barbarino, G. C., Bazilevskaya, G. A., et al. 2013, Advances in Space Research, 51, 219

Aguilar, M., Aisa, D., Alpat, B., et al. 2015a, Phys. Rev. Lett., 114, 171103 Aguilar, M., Aisa, D., Alpat, B., et al. 2015b, Phys. Rev. Lett., 115, 211101 Aharonian, F., Peron, G., Yang, R., Casanova, S., \& Zanin, R. 2018, arXiv eprints, arXiv: 1811.12118

Amato, E. 2014, Internat. Jour. Mod. Phys. D, 23, 1430013

An, Q., Asfandiyarov, R., Azzarello, P., et al. 2019, Science Advances, 5, eaax3793

Atoyan, A. M., Aharonian, F. A., \& Völk, H. J. 1995, Phys. Rev. D, 52, 3265

Atwood, W. B., Abdo, A. A., Ackermann, M., et al. 2009, ApJ, 697, 1071

Baghmanyan, V., Peron, G., Casanova, S., Aharonian, F., \& Zanin, R. 2020, ApJ, 901, L4

Benhabiles-Mezhoud, H., Kiener, J., Tatischeff, V., \& Strong, A. W. 2013, ApJ, 763, 98

Benhabiles-Mezhoud, H., Kiener, J., Thibaud, J. P., et al. 2011, Phys. Rev. C, 83, 024603

Cummings, A. C., Stone, E. C., Heikkila, B. C., et al. 2016, The Astrophysical Journal, 831, 18

D’Angelo, M., Morlino, G., Amato, E., \& Blasi, P. 2018, MNRAS, 474, 1944 de Angelis, A., Tatischeff, V., Grenier, I. A., et al. 2018, Journal of High Energy Astrophysics, 19, 1

Di Mauro, M., Manconi, S., \& Donato, F. 2019, Phys. Rev. D, 100, 123015

Domingo-Santamaría, E. \& Torres, D. F. 2006, A\&A, 448, 613

Han, J. L. 2017, ARA\&A, 55, 111

Indriolo, N., Fields, B. D., \& McCall, B. J. 2009, ApJ, 694, 257

Indriolo, N. \& McCall, B. J. 2012, ApJ, 745, 91

Indriolo, N., Neufeld, D. A., Gerin, M., et al. 2015, ApJ, 800, 40 
Jóhannesson, G., Porter, T. A., \& Moskalenko, I. V. 2018, ApJ, 856, 45

Koning, A., Rochman, D., \& [van der Marck], S. 2014, Nuclear Data Sheets, 118, 187

Koning, A. J., Hilaire, S., \& Duijvestijn, M. C. 2008, in Proceedings of the International Conference on Nuclear Data for Science and Technology, April 2227, 2007, Nice, France, ed. O. Bersillon, F. Gunsing, E. Bauge, R. Jacqmin, \& S. Leray, EDP Sciences, 211-214

Lodders, K. 2010, Astrophysics and Space Science Proceedings, 16, 379

Maestro, P., Adriani, O., Akaike, Y., et al. 2020, Physics of Atomic Nuclei, 82, 766

Malkov, M. A., Diamond, P. H., \& Sagdeev, R. Z. 2011, Nature Communications, 2,194

Mannheim, K. \& Schlickeiser, R. 1994, A\&A, 286, 983

McEnery, J., van der Horst, A., Dominguez, A., et al. 2019, in BAAS, Vol. 51, 245

Murphy, R. J., Kozlovsky, B., Kiener, J., \& Share, G. H. 2009, ApJS, 183, 142

Padovani, M., Galli, D., \& Glassgold, A. E. 2009, Astronomy \& Astrophysics, $501,619-631$

Papadopoulos, P. P. 2010, ApJ, 720, 226

Phan, V. H. M., Morlino, G., \& Gabici, S. 2018, Monthly Notices of the Royal Astronomical Society

Ptuskin, V. 2006, Journal of Physics: Conference Series, 47, 113

Ramaty, R., Kozlovsky, B., \& Lingenfelter, R. E. 1979, ApJS, 40, 487

Sihver, L., Tsao, C. H., Silberberg, R., Kanai, T., \& Barghouty, A. F. 1993, Phys. Rev. C, 47, 1225

Strong, A. W., Moskalenko, I. V., \& Ptuskin, V. S. 2007, Annual Review of Nuclear and Particle Science, 57, 285

Summa, A., Elsässer, D., \& Mannheim, K. 2011, A\&A, 533, A13

Tatischeff, V. \& Kiener, J. 2011, Mem. Soc. Astron. Italiana, 82, 903

Vittino, A., Mertsch, P., Gast, H., \& Schael, S. 2019, Phys. Rev. D, 100, 043007

Weinrich, N., Génolini, Y., Boudaud, M., Derome, L., \& Maurin, D. 2020, A\&A, 639, A131

Yoon, Y. S., Anderson, T., Barrau, A., et al. 2017, ApJ, 839, 5 